\title{
The Recurrence Risk of Ischemic Stroke in Childhood
}

\author{
Vesna Brankovic-Sreckovic ${ }^{a}$ Vedrana Milic-Rasic ${ }^{a}$ Nebojsa Jovic ${ }^{a}$ Natasa Milic ${ }^{b}$ \\ Slobodanka Todorovic ${ }^{\text {a }}$ \\ ${ }^{a}$ Clinic for Child Neurology and Psychiatry and ${ }^{b}$ Institute for Medical Statistics, Medical Faculty, \\ University of Belgrade, Belgrade, Serbia and Montenegro
}

\section{Key Words}

Stroke $\cdot$ Childhood $\cdot$ Recurrence rate

\begin{abstract}
Objective: To determine the risk of recurrence of ischemic stroke in children and to evaluate the influence of etiological factors and underlying mechanisms on recurrence rate. Subjects and Methods: Thirty-six children (21 boys and 15 girls) with clinically and radiographically proven ischemic cerebral infarction were prospectively followed up over a period of $1-9$ years (median 5 years 5 months). The median age of onset of stroke was 8.4 years (1-16 years). Patients with hemorrhagic stroke, neonatal infarction and sinovenous thrombosis were not included. The patients were analyzed according to the mechanisms and etiology of the initial and recurrent stroke event. Results: For the initial stroke, cardioembolic (33.3\%) and arteriopathic processes (36.1\%) were identified as the most probable mechanisms of arterial ischemic stroke. Prothrombotic abnormalities were found in 4 children (11.1\%). Underlying pathology in the remaining 7 (19.4\%) was not known. Recurrent ischemic infarction was diagnosed in 5 children (13.9\%) within 5 days to 18 months (median 6 months) after the first stroke manifestation. In 3 of them stroke recurrence was due to cardiac or transcardiac embolism. Cardiac abnormality prior to the first stroke was detected in 1 child. Clinically silent multiple cerebral infarcts disclosed by MRI preceded the overt stroke episode in 2 patients. Conclusion: Congenital and acquired heart diseases were the most common cause of repeated stroke in
\end{abstract}

\section{KARGER}

Fax +41613061234

E-Mail karger@karger.ch

www. karger.com

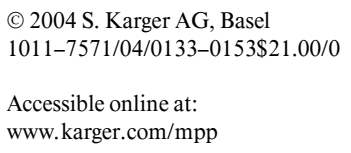

our study. The risk of recurrence appeared to be fivefold higher in children with cardiac disease irrespective of the coexistence of other risk factors. The risk factors of stroke in children were multiple and overlapping. Consequently, recognition of the major one and its underlying mechanism is crucial for both effective therapeutic approach and the prevention of recurrence.

Copyright @ 2004 S. Karger AG, Basel

\section{Introduction}

Arterial ischemic stroke in children is neither a new disorder nor is it as rare as once believed [1]. Although less common than stroke in adults, with an incidence rate of approximately 2.6/100,000/year [2, 3], it is an important cause of lifelong morbidity in childhood and adolescence. Not only are there physical consequences, but also there are profound long-term effects on the child's intellectual, behavioral, and psychosocial functioning and quality of life $[4,5]$. Widespread use of noninvasive and sophisticated diagnostic studies has recently resolved many different aspects of childhood stroke. However, there is still no agreement on classification, outcome, recurrence, or treatment $[3,6]$. Risk factors, which mainly include cardiac abnormalities, vasculopathies, prothrombotic states, infectious and metabolic disorders, are often multiple and overlapping in an individual child, making diagnosis more difficult than on the adult population. Although recognition and awareness of the risk factors for childhood stroke have been improved in the last decade [7], their
Vesna Brankovic-Sreckovic

Clinic for Child Neurology and Psychiatry

Medical Faculty, University of Belgrade

Dr. Subotica 6a, Belgrade (Serbia and Montenegro)

Tel. +38111658 355, Fax +38111645 064, E-Mail vladas@infosky.net 
influence on recurrence rate is unknown, with no randomized controlled trials conducted to date.

Recurrence rate seems to be highly correlated with certain etiologies and underlying mechanisms [8-10]. However, the relevance of these factors to a second ischemic stroke differs from study to study and further research is needed to provide evidence linking these factors to recurrences [9].

The objective of this study was to determine the risk of recurrence in the population of children with arterial ischemic stroke and to identify the relation between the etiology and mechanism of stroke and the recurrence rate.

\section{Subjects and Methods}

\section{Subjects}

Thirty-six children ( 21 boys and 15 girls) with arterial ischemic stroke were prospectively followed up for a median period of 5 years and 5 months, range 1-9 years (January 1, 1993 to January 1, 2002). The median age of the first stroke onset was 8 years and 4 months (ranging from 1 to 16 years). The majority of children were healthy prior to the stroke. The most common clinical presentations of the acute stroke were acute hemiparesis $(\mathrm{n}=32)$ without or with altered consciousness $(n=12)$, motor aphasia/dysphasia $(n=18)$ and seizures $(n=10)$. Eight children had diagnoses of other diseases or abnormalities before the first stroke: surgical closure of foramen ovale in 1, congenital defects (palatoschisis, syndactylia) in 2, febrile and nonfebrile seizures in 2 , migraine in 2 children and bilateral lens subluxation with suspected homocystinuria that was operated upon in 1. Children with hemorrhagic stroke, neonatal infarction and sinovenous thrombosis were excluded from the study. The diagnosis was established by the strict clinical and radiographic criteria that included acute focal neurological dysfunction lasting more than $24 \mathrm{~h}$ and a computed tomography (CT scan) and/or magnetic resonance imaging (MRI) of the brain showing focal infarction in the vascular territory corresponding with the neurological presentation.

\section{Clinical and Diagnostic Information}

The information included age, gender, familial and personal medical history, and clinical features. Neurological deficits were described by their type and severity at the time of admission and after 1, 3 and 6 months consecutively, followed by annual assessment during the enrollment period. Outcome was estimated using the Pediatric Stroke Outcome Measure 3 and 6 months after stroke [11]. It was considered as (a) favorable if the patient's outcome was completely normal (scored 0 ) or with mild deficit (scored 0.5 ) or (b) unfavorable if a neurological deficit was moderate (scored 1) or severe (scored 2). Definition of these categories consisted of the presence or absence of deficits in five spheres: right sensorimotor, left sensorimotor, language expression, language comprehension and cognitive and behavioral. Diagnostic studies were preceded using step-by-step analyses. All patients underwent routine blood tests and a coagulation study, including protein $\mathrm{C}$, protein $\mathrm{S}$, antithrombin III, lupus anticoagulant and anticardiolipin. For technical reasons, genetic tests for factor V Leiden were not performed. Chest radiography, electrocardiography, transthoracic echocardiography, electroenceph- alography and at least one of neuroimaging and vascular imaging studies (CT scan, MRI, magnetic resonance angiography (MRA), or catheter angiography) were performed as part of the ongoing clinical investigation. Intra-arterial catheter cerebral angiography was done in 25 patients, while MRA was used either in patients $(n=10)$ in whom contrast angiography was contraindicated, or during the clinical follow-up. Carotid and/or transcranial ultrasonography was done in 15 patients. Transesophageal echocardiography (TEE) was recorded in selected cases $(n=7)$. A recurrent cerebrovascular event was considered if a new stroke, defined as: acute occurrence of focal neurological deficit lasting more than $24 \mathrm{~h}$, or worsening of an existing deficit accompanied by a new lesion on neuroimaging, or recurrent transient ischemic attacks (TIAs) in moyamoya disease, were documented.

\section{Classification of Stroke Subtypes by Mechanism}

Acute arterial ischemic stroke was classified into five types: (a) cardiac stroke (congenital and acquired heart disease, rhythm disturbances); (b) vascular or arteriopathic stroke (vasculopathies, vasculitis, arterial hypoplasia, fibromuscular dysplasia, arterial dissection, postvaricella arteriopathy); (c) stroke associated with infectious diseases; (d) stroke associated with prothrombotic abnormalities, and (e) idiopathic stroke (no obvious cause of stroke or associated conditions) $[7,12]$.

\section{Statistical Analyses}

The results are expressed as means with standard deviation and percentages where appropriate. Two-by-two tables were constructed to analyze the intergroup relations. Significance level was tested using the chi-square $\left(\chi^{2}\right)$ test and Fisher's exact test. The level of significance for testing all null hypotheses was a two-tailed $\mathrm{p}<0.05$. Relative risk (RR) and confidence intervals $(95 \% \mathrm{CI})$ were calculated for the risk factors.

\section{Results}

The initial types of stroke and recurrence rates are listed in table 1. Cardiac or transcardiac embolic stroke occurred in $12(33.3 \%)$, and vascular, prothrombotic and idiopathic strokes occurred in $13(36.1 \%), 4(11.1 \%)$ and $7(19.4 \%)$, respectively. In the cardiac or transcardiac embolic group 3 children had infectious disease (acute respiratory, gastrointestinal and Borrelia infections) that was detected prior to or at the time of embolic stroke. The embolism was suspected in 2 of them with streptococcal and Borrelia infections and was considered as the underlying mechanism of the stroke based on both clinical and imaging characteristics of infarction, even though cardiac abnormality was not obvious.

Clinically silent cerebral infarcts disclosed by MRI preceded the overt stroke episode in 2 patients. In one 15year-old child (fig. 1a, b) multiple infarcts restricted to the right hemisphere as well as cardiomyopathy and rhythm abnormalities in the form of Wolff-Parkinson-White (WPW) syndrome, and polycystic kidney disease were 

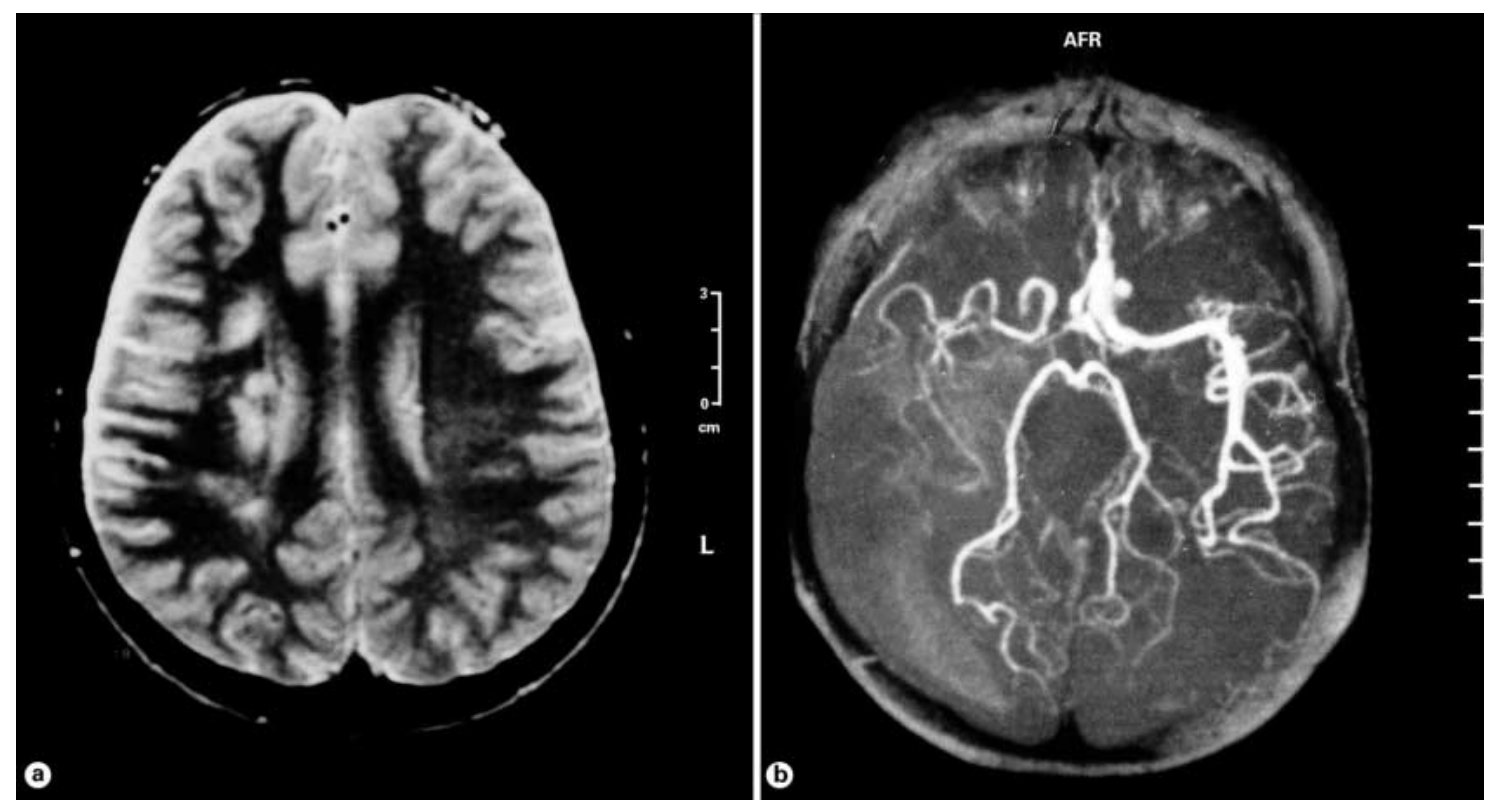

Fig. 1. a $T_{2}$-weighted $M R I$ of the brain showing old, lacunar multiple subcortical infarctions and the new large cortical-subcortical infarction in the right hemisphere in a child with cardiomyopathy and WPW syndrome. b MRA revealing occlusion of the right middle cerebral artery.

Table 1. Stroke subtypes according to mechanism and recurrence rate of arterial ischemic stroke in 36 children (9-year follow-up)

\begin{tabular}{|c|c|c|}
\hline Mechanism & $\begin{array}{l}\text { Patients } \\
(\mathrm{n}=36)\end{array}$ & $\begin{array}{l}\text { Recurrence } \\
\text { of stroke }\end{array}$ \\
\hline Cardiac or transcardiac embolism ${ }^{\mathrm{a}}$ & $12(33.3 \%)$ & $3 / 12(25 \%)^{\mathrm{b}}$ \\
\hline Congenital cardiac diseases & 7 & \\
\hline Acquired cardiac diseases & 3 & \\
\hline Infectious diseases ${ }^{\mathrm{a}}$ & 2 & \\
\hline Vascular stroke due to & $13(36.1 \%)$ & $2 / 13(15.4 \%)$ \\
\hline Transient arteriopathies & 3 & \\
\hline Structural vascular abnormalities & 2 & \\
\hline Moyamoya disease & 2 & \\
\hline Migraine & 2 & \\
\hline Vasculitis & 1 & \\
\hline Homocystinuria & 1 & \\
\hline Postvaricella angiopathy & 1 & \\
\hline Fibromuscular dysplasia & 1 & \\
\hline Stroke associated with prothrombotic states & $4(11.1 \%)$ & $0 / 4(0 \%)$ \\
\hline Protein $\mathrm{C}$ deficiency & 1 & \\
\hline Anemia/secondary thrombocytosis & 1 & \\
\hline Antiphospholipid antibodies syndrome & 1 & \\
\hline Hypercholesterolemia & 1 & \\
\hline Idiopathic stroke & $7(19.4 \%)$ & $0 / 7(0 \%)$ \\
\hline
\end{tabular}

a Including 2 patients with coexisting infectious disease (streptococcal infection and Borrelia infection).

b Fisher's exact test: $\mathrm{p}=0.029, \mathrm{p}<0.05$. 

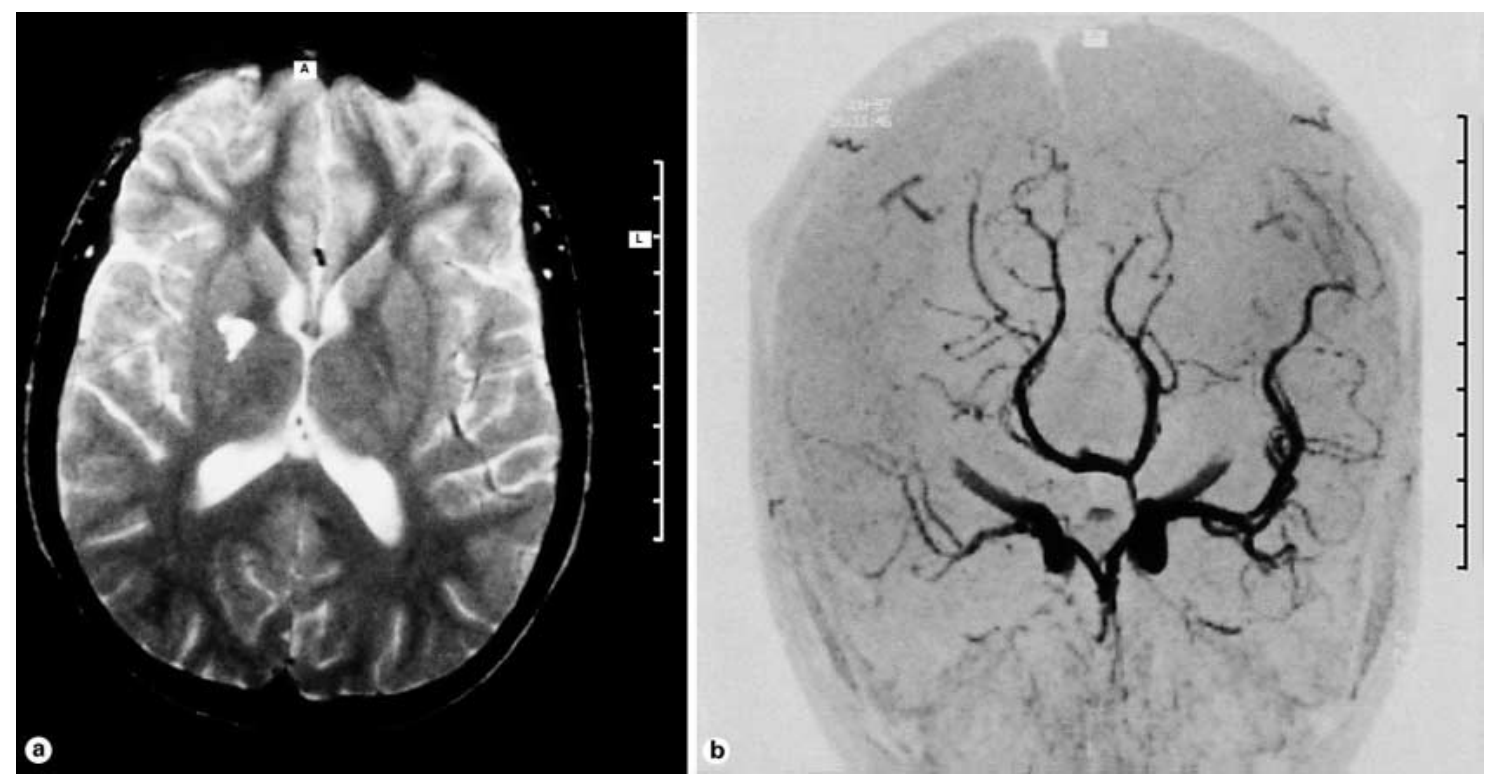

Fig. 2. MRI and MRA of a 14-year-old patient with migraine-related stroke and repeated TIAs. a Infarctions in the right putamen, globus pallidus, and capsular region. b Subocclusion of the M2 segment of the right middle cerebral artery and hypoplasia of the right posterior communicating artery.

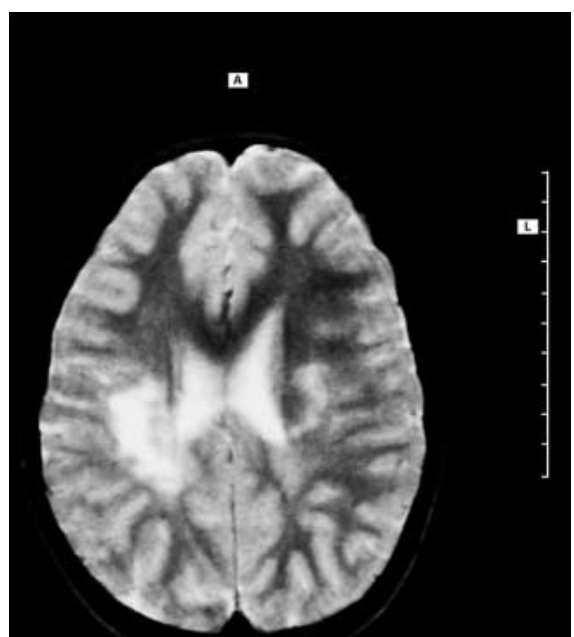

Fig. 3. $T_{2}$-weighted MRI of the brain showing left- and right-sided subcortical infarctions in a 6-year-old boy with patent foramen ovale and gastrointestinal infection.

diagnosed at the time of evident stroke. Mitral valve prolapse and endovascular closure of patent foramen ovale were documented 2 years before the overt stroke in another 3-year-old child with multiple infarcts in the left hemisphere. Migraine-related stroke was diagnosed in a 14year-old boy (fig. 2a, b). He had a history of migraine with unilateral sensorimotor aura (being considered as re- peated TIAs lasting several hours and occurring once annually, since he was 8).

Seven (19.4\%) of 36 children with ischemic stroke had no residual impairment. Functional outcome was good in 15 children (41.7\%) and poor in $21(58.3 \%)$ with moderate deficit persisting in $27.8 \%$ and severe in $30.5 \%$.

Recurrent ischemic infarction was diagnosed in 5 (13.9\%) children within 5 days to 18 months (median 6 months) after the manifestation of the first stroke. In 3 of them the stroke recurrence was due to cardiac or transcardiac embolism, while the in the remaining 2 it was due to vascular stroke. There was statistically significant intergroup difference between the recurrent stroke rate in children with (including 2 patients with multiple infarction prior to the manifested stroke) and without cardiac disease $(p=0.029)$.

In a 6-year-old boy (patient 1), pulmonary artery stenosis and persistent left-sided superior vena cava were diagnosed before the first stroke. Repeated infarction by embolic mechanism was diagnosed 12 and 18 months, respectively, after the first stroke episode. Iron deficiency anemia and pericardial exudation were additionally diagnosed during the third stroke episode. CT and MRI of the brain disclosed multiple subcortical infarctions in different stages, the newest corresponding with persistent neurological deficit. A moderate right hemiparesis and cognitive impairment were found during the clinical follow-up. 
Table 2. Localizations of initial and recurrent strokes according to the etiology

\begin{tabular}{|c|c|c|c|}
\hline \multirow[t]{2}{*}{ Patient } & \multirow[t]{2}{*}{ Risk factors } & \multicolumn{2}{|l|}{ Localization } \\
\hline & & initial stroke & recurrent stroke \\
\hline 1 & Congenital heart disease, hypochromic anemia & right-sided FT subcortical region & $\begin{array}{l}\text { lacunar infarctions in the right capsular and left } \\
\text { caudate nucleus }\end{array}$ \\
\hline 2 & Patent foramen ovale, echoviral infection & cortical-subcortical FPT left-sided infarction & subcortical FT right-sided infarction \\
\hline 3 & Patent foramen ovale & subcortical FT left-sided infarction & right lacunar infarction of basal ganglia \\
\hline 4 & Dilatative cardiomyopathy, WPW syndrome & multiple subcortical right-sided infarction & cortical-subcortical FT right-sided infarction \\
\hline 5 & Congenital heart disease, cardiac surgery & left basal ganglia infarction & left temporal region \\
\hline 6 & Moyamoya disease & right capsulothalamic region & right caudate nucleus \\
\hline 7 & Homocystinuria & left subcortical PT infarction & left-sided FPT cortical-subcortical infarction \\
\hline
\end{tabular}

Patients 4 and 5 presented with clinically silent infarction revealed by MRI at the time of manifested stroke. FT = Frontotemporal; FPT $=$ frontoparietotemporal; $\mathrm{PT}=$ parietotemporal.

In the other 2 patients, 6- (fig. 3) and 7-year-old boys (patients 2 and 3, respectively), the second stroke occurred within 10 days after the first cerebral infarction. In these children patent foramen ovale, not recommended for endovascular surgical procedure, was diagnosed after the first stroke. In the 6-year-old boy, gastrointestinal infection with raised echoviral titer was detected a few days prior to the first stroke. Both children had moderate residual motor deficits during the 5-year follow-up period. All 3 children with suspected cardiac embolism received antiaggregation therapy (aspirin $2-3 \mathrm{mg} / \mathrm{kg} /$ day) at the time of the second stroke.

In patients with 'vascular stroke etiology', repeated TIAs affecting the same arterial territory in 1 patient with moyamoya disease, and recurrent stroke in a child with homocystinuria were diagnosed. Conventional angiography revealed the moyamoya pattern in a 13-year-old girl after repeated transient hemiparesis and seizures. Repeated strokes within $48 \mathrm{~h}$ in the 14-year-old girl with homocystinuria occurred 1 month after surgical treatment of lens subluxation. The locations of the ischemic lesions, presented as the initial and recurrent strokes, according to the etiology, are illustrated in table 2. All the children with 1 or more recurrent strokes had a poor outcome. None of them had seizures as sequelae. Death occurred in the patient with homocystinuria as a result of stroke-associated complications (raised intracranial pressure and refractory status epilepticus).

\section{Discussion}

Although stroke in childhood occurs less frequently than in the adult population, consequences may be devastating for the child's and family's life [13]. The etiological diversity, with a wide array of risk factors in children, seems to be the major difference between pediatric and adult stroke. Even more, the risk factors are often multiple and overlapping, making an etiological diagnosis more challenging [14]. The exact influence of each factor on the mechanism of stroke is difficult to establish in an individual child. Despite extensive evaluations, an etiology could not be defined in up to one third of children [15]. The most common risk factors for stroke in children are congenital and acquired heart disease [3,8], progressive (moyamoya, vasculitis) and transient arteriopathies [12], sickle cell disease, prothrombotic abnormalities (protein $\mathrm{C}$, protein $\mathrm{S}$, factor V Leiden) [16], metabolic and infectious diseases [17] and minor head trauma [18]. It has been emphasized that both the outcome and risk of recurrences depend on the etiology and mechanism of the stroke $[9,12]$ as well as on the coexistence of multiple risk factors $[8,14]$. Congenital and acquired cardiac disease $[2,3,19]$ and sickle cell disease [20] have been pointed out as predictors for recurrent ischemic stroke. Despite well-known and recognizable complex congenital heart defects, more subtle abnormalities such as minimal mitral valve prolapse, patent foramen ovale and atrial septal aneurysm have been identified as potential risk factors for recurrent stroke in children and young adults [9, 21]. Additionally, in some instances these conditions can be considered as hereditary. Idiopathic autosomal dominant mitral valve prolapse per se and as a part of connective tissue disease, hereditary cardiomyopathies, conduction abnormalities (including WPW syndrome), and cardiac abnormalities in mitochondrial disease $[19,22]$ are recognized as genetically determined conditions. Recently, the relevance of other factors to the second stroke has been reported, including prothrombotic abnormalities [10], arterial dissection [23], transient arteriopathies and varicella-associated stroke [24]. 
The highest proportion of recurrent stroke (60\%) in our study was found in children with underlying heart disease, which suggested that cardiac and transcardiac embolism were the most common mechanisms of repeated cerebral infarction. After inclusion of 2 patients with clinically silent infarctions disclosed by MRI, in whom a cardiac abnormality was detected at the time of manifested stroke, this proportion is even higher with a relative risk rate of $\mathrm{RR}=5.25 ; 95 \%$ CI 1.35-20.45. Furthermore, 2 previously healthy children with no history of cardiac disease were diagnosed with patent foramen ovale using TEE. That suggests that TEE is a useful tool for detecting an intracardiac source of embolism [25]. In addition, infectious diseases are frequent in children. They may be a trigger for a transient hypercoagulable state which, with a coexisting minor cardiac abnormality (i.e. patent foramen ovale), could lead to paradoxical emboli and cerebral infarction [26].

Two children without previously diagnosed cardiac abnormalities received antiaggregation therapy for a short time (5-7 days) between the first and the second stroke, whereas patient 1 with known cardiac disease had been receiving aspirin intermittently over a period of 1 year. The advantage of the antiaggregation therapy (aspirin) was difficult to assess due to the short-term treatment (patients 2 and 3) and poor compliance (patient 1). Other therapeutic regimes have been suggested and should be considered in those children who are at a higher risk of stroke recurrence [27].

\section{Conclusion}

Identification of the underlying condition and mechanism of stroke is important and can be of certain predictive value for both outcome and recurrence rate. Because of the overlapping of multiple risk factors in a number of children with stroke, a cardiac source of embolism may be overlooked. As such, even in the absence of any clinical stigmata, children with stroke should be evaluated for cardiac disease. Appropriate management of these risk factors may prevent and reduce stroke recurrence.

\section{References}

1 Osler W: The Cerebral Palsies of Children: A Clinical Study from the Infirmary of Nervous Disease. Philadelphia, Blakiston, 1989, pp 155.

2 Schoenberg BS, Mellinger JF, Schoenberg DG: Cerebrovascular disease in infant and children: A study of incidence, clinical features, and survival. Neurology 1978;28:763-768.

3 deVeber G, Roach SE, Riela AR, Wiznitzer M: Stroke in Children: Recognition, Treatment, and Future Directions. Semin Pediatr Neurol 2000;7:309-317.

4 Goodman R: Psychological aspects of hemiplegia. Arch Dis Child 1997;76:177-178.

5 Ganesan V, Hogan A, Shack N, Gordon A, Isaacs E, Kirkham FJ: Outcome after ischemic stroke in childhood. Dev Med Child Neurol 2000;42:455-461.

6 Lynch JK, Hirtz DG, deVeber G, Nelson KB: Report of the National Institute of Neurological Disorders and Stroke Workshop on perinatal and childhood stroke. Pediatrics 2002;109: 116-123.

7 Kirkham FJ, Prengler M, Hewes DK, Ganesan $\mathrm{V}$ : Risk factors for arterial ischemic stroke in children. J Child Neurol 2000;15:299-307.

8 Roach ES: Etiology of stroke in children. Semin Pediatr Neurol 2000; 7:244-260.

9 Mas JL, Arquizan C, Lamy C, Zuber M, Cabanes L, Derumeaux G, Coste J: Recurrent cerebrovascular events associated with patent foramen ovale, atrial septal aneurysm, or both. N Engl J Med 2001;345:1740-1746.

10 Strater R, Becker S, von Eckardstien A, Heinecke A, Gutsche S, Junker R, Kurnik K, Schobess R, Nowak-Gottl U: Prospective assess- ment of risk factors for recurrent stroke during childhood: A 5-year follow-up study. Lancet 2002;360:1540-1545.

11 deVeber GA, MacGregor D, Curtis R, Mayank $\mathrm{S}$ : Neurologic outcome in survivors of childhood arterial ischemic stroke and sinovenous thrombosis. J Child Neurol 2000;15:316-324.

12 Chabrier S, Husson B, Lasjaunias P, Landrieu $\mathrm{P}$, Tardieu M: Stroke in childhood: Outcome and recurrence risk by mechanism in 59 patients. J Child Neurol 2000;15:290-294.

13 Gordon AL, Ganesan V, Towell A, Kirkham FJ: Functional outcome following stroke in children. J Child Neurol 2002;17:429-434.

14 Lanthier S, Carmant L, David M, Larbrisseau A, deVeber G: Stroke in children: The coexistence of multiple risk factors predicts poor outcome. Neurology 2000;54:371-378.

15 Kirkham FJ: Stroke in childhood. Arch Dis Child 1999;81:85-89.

16 Kenet G, Sadetzki S, Murad H, Martinowitz U, Rosenberg N, Gitel S, Rechavi G, Inbal A: Factor V Leiden and antiphospholipid antibodies are significant risk factors for ischemic stroke in children. Stroke 2000;31:1283-1288.

17 Martinez-Fernandez E, Gil-Peralta A, GarciaLozano R, Chinchon I, Aguilera I, FernandezLopez O, Arenas J, Campos Y, Bautista J: Mitochondrial disease and stroke. Stroke 2001; 32:2507-2510.

18 Kieslich M, Fiedler A, Heller C, Kreuz W, Jacobi G: Minor head injury as cause and cofactor in the aetiology of stroke in childhood: A report of eight cases. J Neurol Neurosurg Psychiatry 2002;73:13-16.
19 Pavlakis SG, Gould RJ, Zito JL: Stroke in children. Adv Pediatr 1991;38:151-179.

20 Pegelow CH, Adams RJ, McKie V, Abboud M, Berman B, Miller ST, Olivieri N, Vichinsky E, Wang W, Brambilla D: Risk of recurrent stroke in patients with sickle cell disease treated with erythrocyte transfusions. J Pediatr 1995;126: 896-899.

21 McGaw D, Harper R: Patent foramen ovale and cryptogenic cerebral infarction. Intern Med J 2001;31:42-47.

22 Hassan A, Markus HS: Genetics and ischaemic stroke. Brain 2000;123:1784-1812.

23 Fullerton HJ, Johnston CS, Smith WS: Arterial dissection and stroke in children. Neurology 2001;57:1155-1160.

24 Askalan R, Laughlin S, Mayank S, Chan A, MacGregor D, Andrew M, Curtis R, Meaney B, deVeber G: Chickenpox and stroke in childhood: A study of frequency and causation. Stroke 2001;32:1257-1262.

25 Strandberg M, Marttila RJ, Helenius H, Hartiala J: Transoesophageal echocardiography in selecting patients for anticoagulation after ischemic stroke or transient ischemic attack. J Neurol Neurosurg Psychiatry 2002;73:29-33.

26 Higgins JJ, Kammerman LA, Fitz CR: Predictors of survival and characteristics of childhood stroke. Neuropediatrics 1991;22:190-193.

27 Strater R, Kurnik K, Heller C, Schobess R, Luigs P, Nowak-Gottl U: Aspirin versus lowdose low-molecular-weight heparin: Antithrombotic therapy in pediatric ischemic stroke patients - a prospective follow-up study. Stroke 2001;32:2554-2558. 